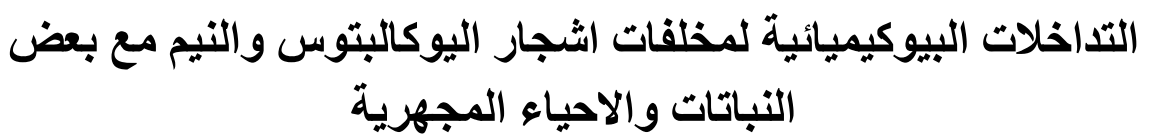

\title{
Biochemical interaction between Eucalyptus, Neem residues with some plants and microorganisms
}

نهاد طه محمد

انسام غازي العاني

A.W.AL-SHAHWANY A. G. AL -AANI N. T. MOHAMMAD

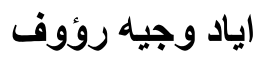

كلية العلوم / جامعة بغداد

College of Science/ University of Baghdad

المستخلص

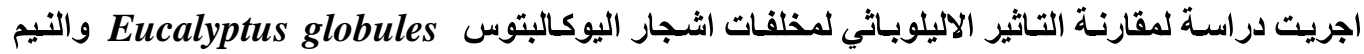

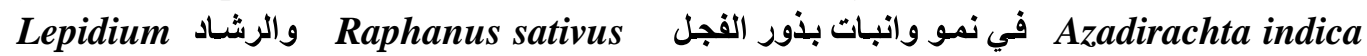

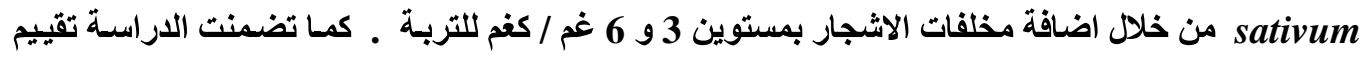
الفعالية الميكروبية لزيت بذور اليوكالبتوس والنيم على الاحياء المجهريـة الممرضة و Pseudomonus aeruginosa و Staphylococcus aureusiella pnenumonia ال Proteus mirabilis

البخاري Stem Distillation في استخلاص الزيوت ، كما اعتمدت طريقة

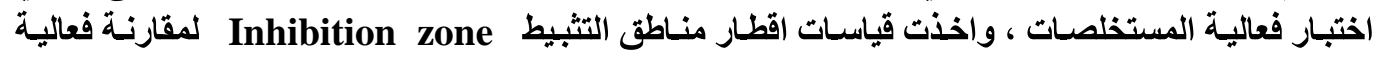

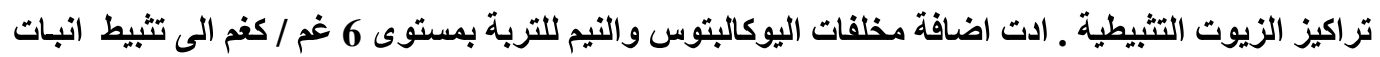

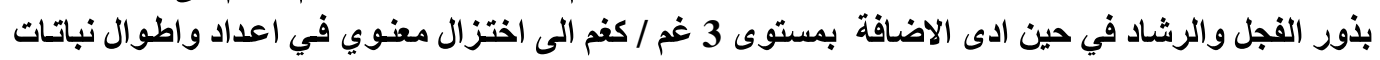

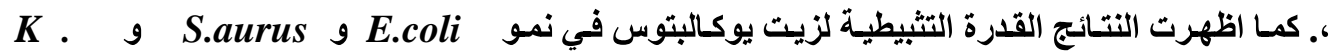

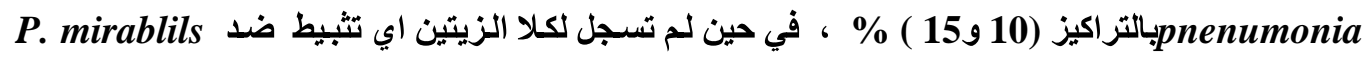

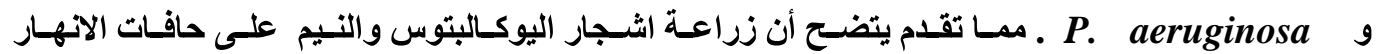

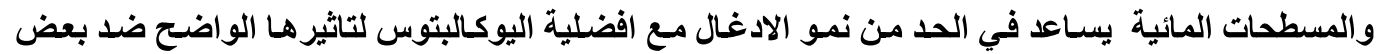

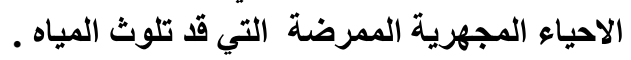

\section{Abstract}

The study was carried to determinate the allelopathic effects of Eucalyptus globules and Azadirachta indica against Raphanus sativus and Lepidium sativum by adding their residues to the soil at rates of $(3,6) \mathrm{gr} / \mathrm{kg}$ soil . The second part of study was to extract the oil seed and determine the biological activities against some pathogenic microorganisms namely Escherichia col, Klebesiella pneumonia, Staphylococcus aureus, Pseudomonas aeruginosa, Proteus mirabilis. These oils used at cons. $(2.5,5,10,15) \%$ prepared and separated by stem distillation. Agar well diffusion method was used in bacteria preparing, and comparing extracts effect by determining the inhibition zone. Residues of Eucalyptus and Neem added to the soil at rates of $3 \mathrm{gr} / \mathrm{kg}$ soil significantly reduced the seed germination number and shoot length. While adding $6 \mathrm{gr} / \mathrm{kg}$ soil inhibited the seedling growth. Also the result shows inhibitory effects of the 
Eucalyptus oils against $E$.coli, $S$. aurus and $K$. pnenumonia, put there was no inhibition against $P$. aeruginosa and $P$.mirablils treatments. In conclusion, it seem much useful to cultivated Eucalyptus and Neem trees at river bank and lake beach because their residues could be use as herbicides, antibacterial against same microorganisms which polluted the water.

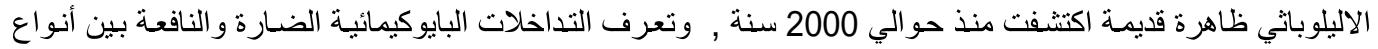

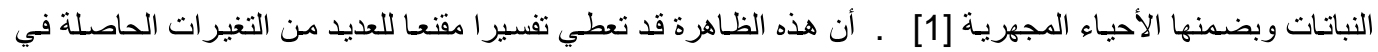

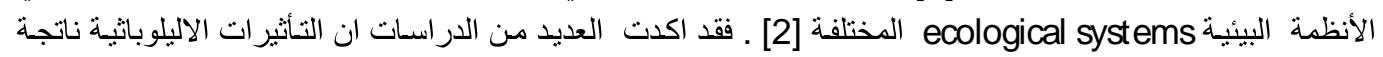

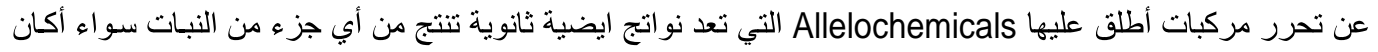

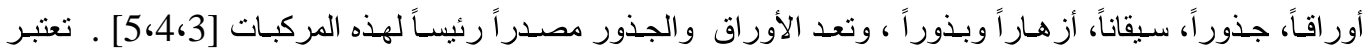

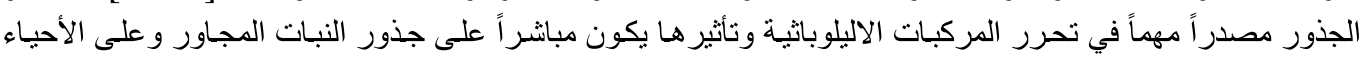

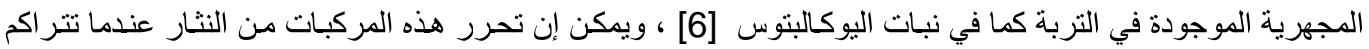

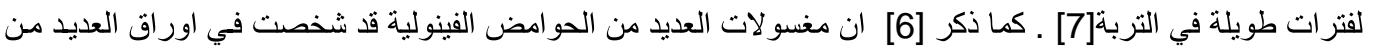

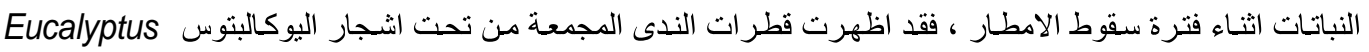
globules L

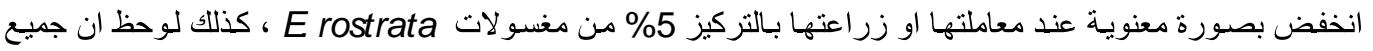

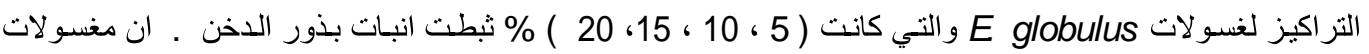

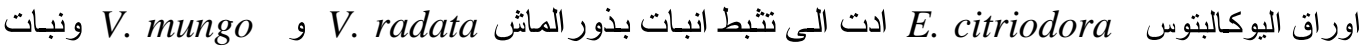
Arachis hypogaea

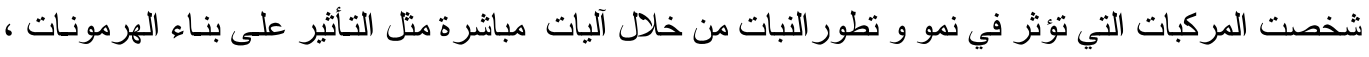

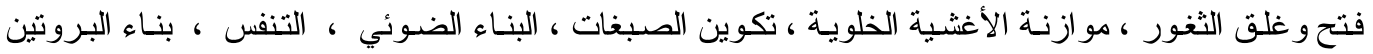

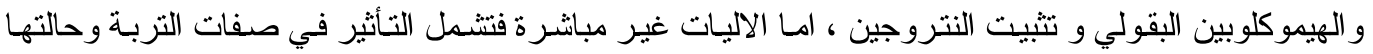

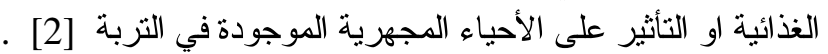

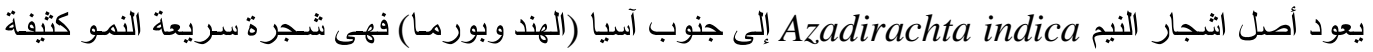

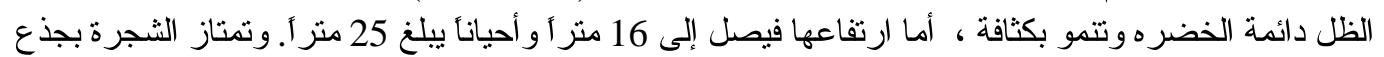

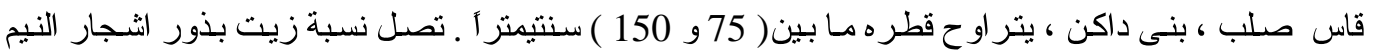

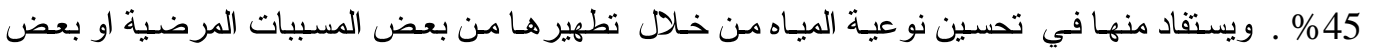

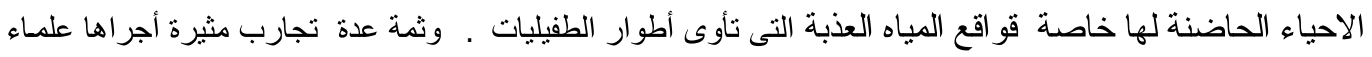

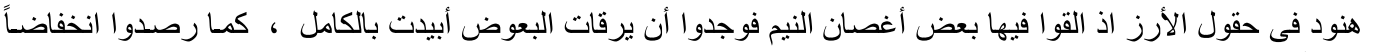

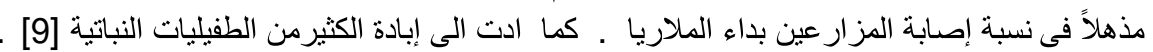

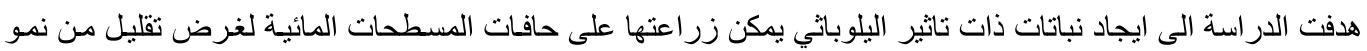

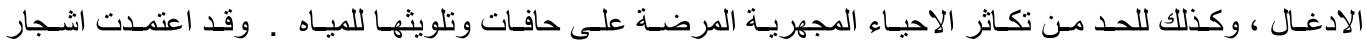
اليوكالبتوس و النيم لسر عة نمو هما ولفو ائدهما الاقصادية فضلا عن الستخدامات الطبية لهما .

المواد وطرائق البحث

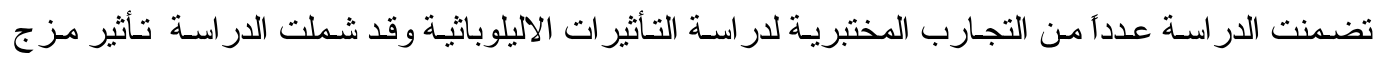

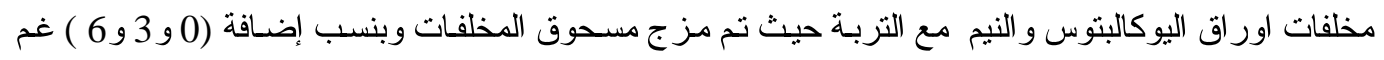

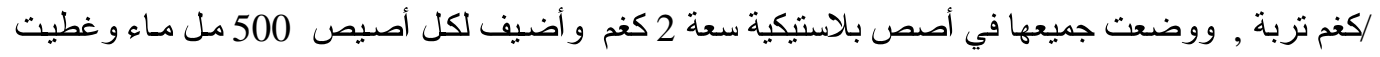

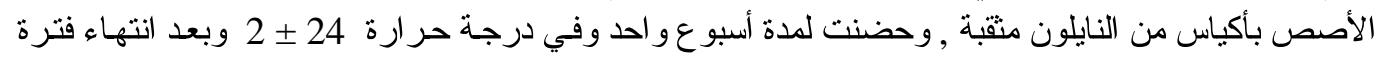

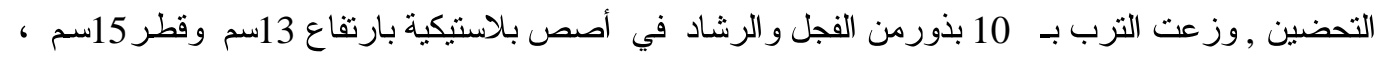

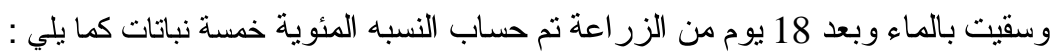




$$
\begin{aligned}
& \text { النسبة المئوية للإنبات } \\
& \text { كما سجلت اعداد و اطو ال تللك النباتات . }
\end{aligned}
$$

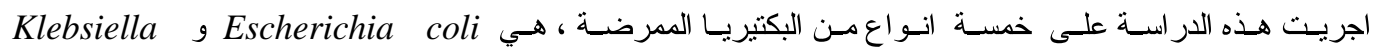
Proteus mirabilis و Pseudomonus aeruginosa gtaphylococcus aureus gneuumonia المستحصل عليها من مختبر ات الاحياء المجهرية للار اسات العليا في قسم علوم الحياة لكلية العلوم - جامعة بغداد و التي تم

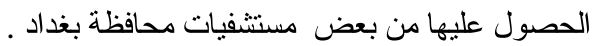
تم تجفيف البذور على درجـة حـر ارة 35 م لمـدة 24 سـاعة بعد ذللك طحنـت البذور بو اسـطة مطحنـة كهربائيسة .

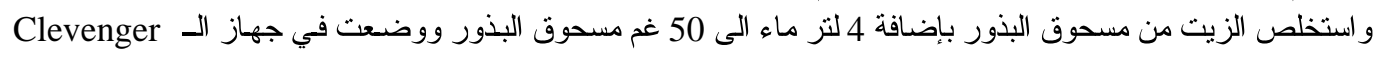
حيث اتبعت طريقة التقطير البخاري Steam Distillation في الحصول على الزيوت ، تم تخفيف الزيت بتر اكيز ( 0 و و 5 و 10 و 15) باستعمال اثلين كلايكول .

\section{تحضير المزروع البكتيري : تحضير}

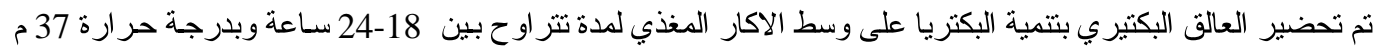

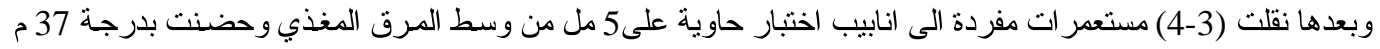

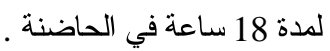
تاثير التراكيز المختلفة لزيت بذور في نمو الاحياء المجهرية الممرضة :

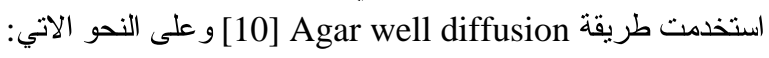
• صب 25 مل من وسط الاكار المغذي لكل طبق زجاجي . تمت مجانسة العالق البكتيري ذي زمن 18 ساعة ، و واجريت له التخافيف العشرية . لقح الوسط بـ 0.1 مللتير من العالق البكنيري ذي ثركيز 1 × 10 10 وحدة مكونة للمستعدرة / مل ونشئر

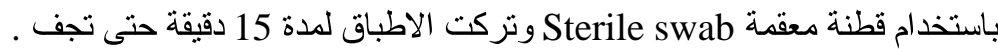

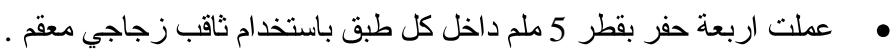

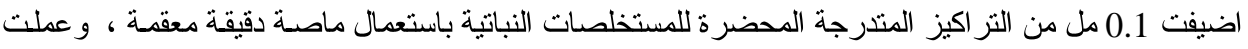

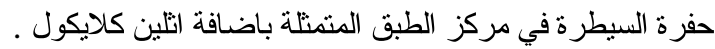
عملت ثلاثة مكرر ات لكل طبق ، بعدها حضنت الاطباق بدرجة 37 م لمدة 24 ساعة في الحاضنة .

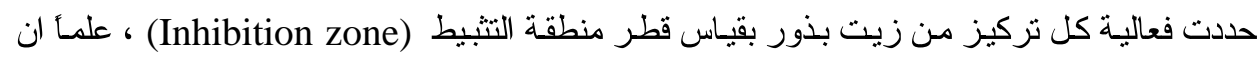

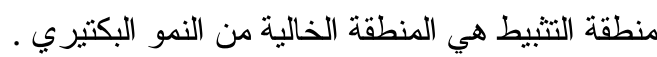

نفذت التجـارب حسـب تصـميم القطاعـات العشـو ائية الكاملـة(R.C.B.D) ، اجـري التحليل الإحصسائي للبيانـات باستخدام الحاسـوب الآلي بو اسطة برنـامج SAS ومقارنـة المتوسطات الحسـابية باستعمال اختبـار دنكن متعدد المدى .

النتائج والمناقشة 1. تاثير اضافة مخلفات اليوكالبتوس والتيم في انبات ونمو بذور الفجل والرشاد

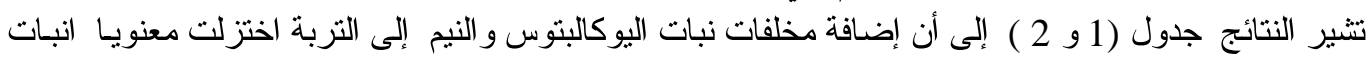

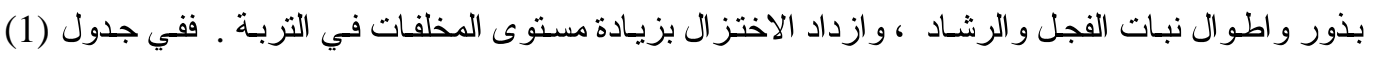

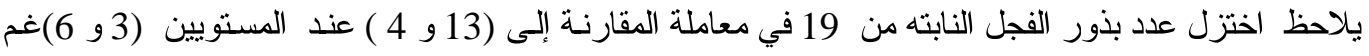

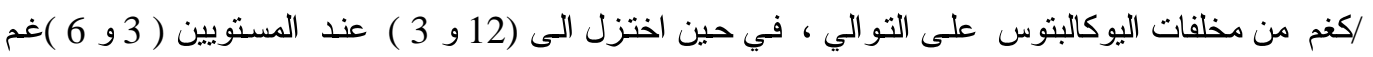
/كغم عند استخدام مخلفات النيم على التو الي ـ امـابذور الرشـاد فقد اختزل اعداد البذور النابته من من 14 في

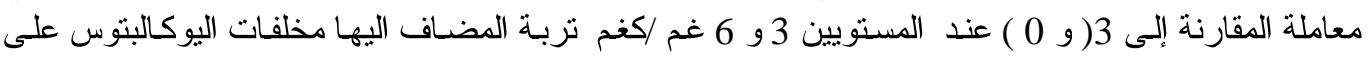

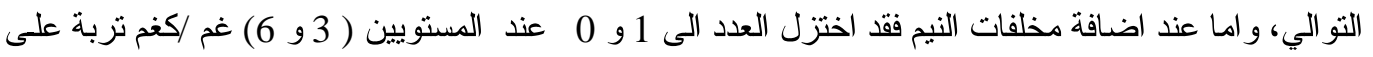
التو الي ـ كما اشارة النتائج الى تفوق نبات النيم بتاثير اته الاليلوباثيه. 
جدول (2): معدل اعداد البذور الفجل والرشاد النابتة

\begin{tabular}{|c|c|c|c|c|c|c|}
\hline \multicolumn{2}{|c|}{ اعداد البذور النابتة في تربة ممزوجة 6 غم اكفم تربة } & \multicolumn{2}{|c|}{ ممزوجة بمخلفات 3 غم اكغم تربة البذة فئة } & \multirow[t]{2}{*}{ السيطرة } & \multirow[t]{2}{*}{ 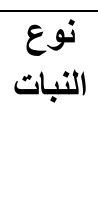 } & \multirow{2}{*}{ E. } \\
\hline نبات التيم & $\begin{array}{c}\text { اليوكالبتوس } \\
\text { البات }\end{array}$ & نبات النيم & نبات اليوكالبتوس & & & \\
\hline $3 c$ & $4 \mathrm{c}$ & $12 \mathrm{~b}$ & $13 \mathrm{~b}$ & $19 \mathrm{a}$ & الفجل & 1 \\
\hline 0 & 0 & $1 \mathrm{~d}$ & $3 \mathrm{c}$ & $14 \mathrm{~b}$ & الرشاد & 2 \\
\hline
\end{tabular}

و اختزلت مخلفات اليوكالبتوس و النيم معنويا اطو ال النباتات النامية و ازداد الاختز ال بزيادة مسنتوى المخلفات .

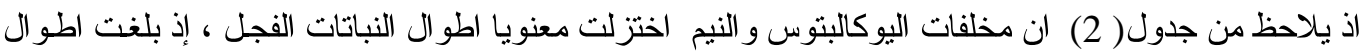

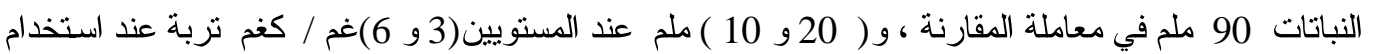

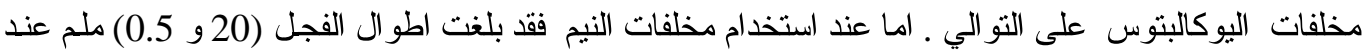

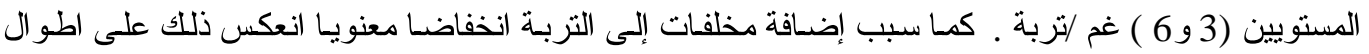

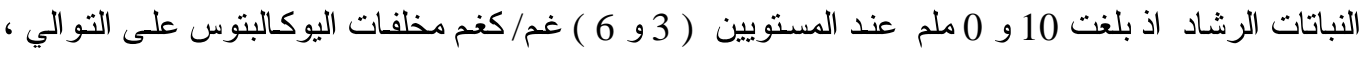

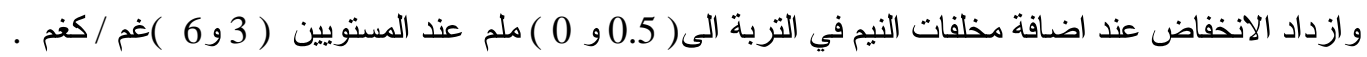

جدول (2) : معدل اطوال نباتات الفجل و الرشاد ( ملم )

\begin{tabular}{|c|c|c|c|c|c|c|}
\hline \multicolumn{2}{|c|}{ مزوجة بمخلفات } & \multicolumn{2}{|c|}{ ممزوجة بمخلفات 3 غم اكفئة } & \multirow[t]{2}{*}{ السيطرة } & \multirow[t]{2}{*}{ التبات } & \\
\hline نبات التيم & نبات اليوكالبتوس & نبات النيم & نبات اليوكالبتوس & & & \\
\hline $0.5 \mathrm{c}$ & $10 \mathrm{c}$ & $20 \mathrm{~b}$ & $20 \mathrm{~b}$ & $90 \mathrm{a}$ & الفجل & 1 \\
\hline 0 & 0 & $0.5 \mathrm{~d}$ & $10 \mathrm{c}$ & $30 \mathrm{~b}$ & الرشاد & 2 \\
\hline
\end{tabular}

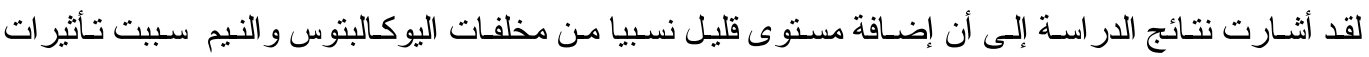

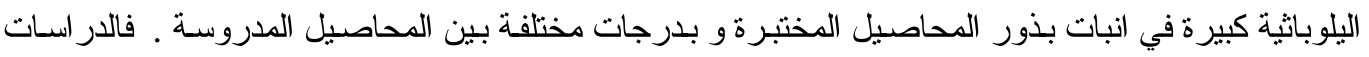

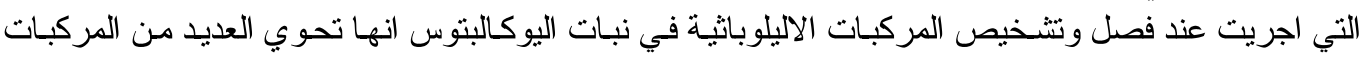

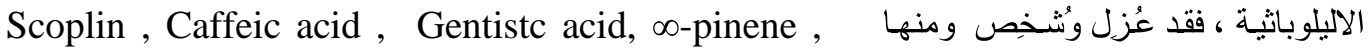
Chlorogenic acid, Ferulic acid ,Camphore, P-coumaric

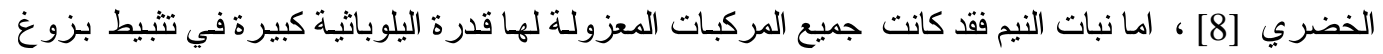

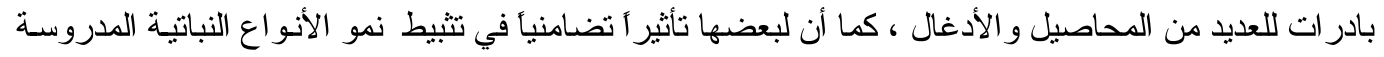

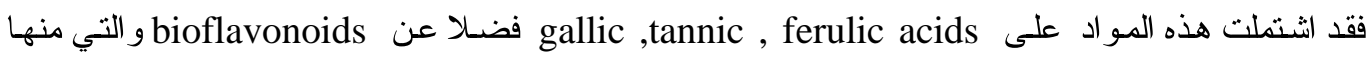

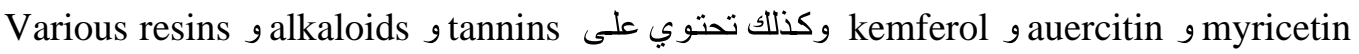

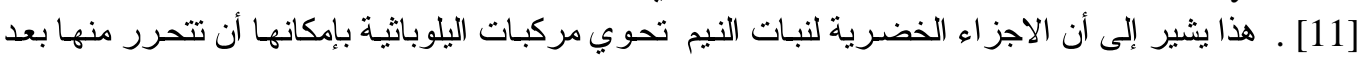

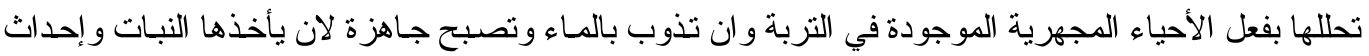

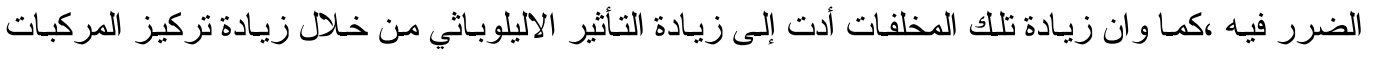

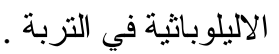

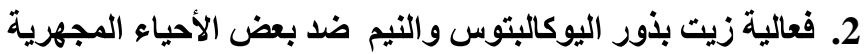

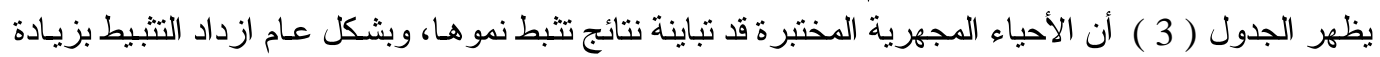

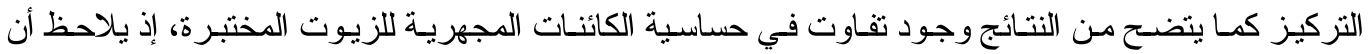

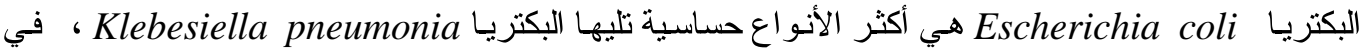

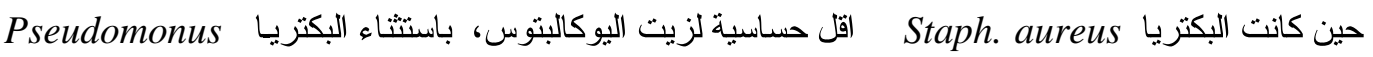


Proteus mirabilis التي لم "ثثبط بكلا الزيتين ـويلاحظ أيضـا أن زيت النيم لم يكن مثبطا بشكل و اضتح حتى عند زيادة التز اكيز المستخدمة .

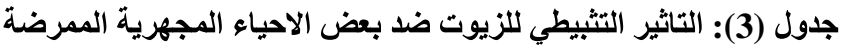

\begin{tabular}{|c|c|c|c|c|c|c|c|c|c|c|c|c|c|c|c|c|c|c|c|c|}
\hline \multicolumn{21}{|c|}{ حقول التثبط ( ملم ) Zone of inhibition (mm) } \\
\hline \multicolumn{4}{|c|}{ S. aureas } & \multicolumn{4}{|c|}{ P. mirabils } & \multicolumn{4}{|c|}{ P. aeruginosa } & \multicolumn{4}{|c|}{ Klebsiella sp. } & \multicolumn{4}{|c|}{ E. coli } & \\
\hline$\frac{1}{20}$ & $\frac{2}{2}$ & $\begin{array}{l}10 \\
8\end{array}$ & in & $\frac{10}{6}$ & $\frac{0}{2}$ & $\begin{array}{l}10 \\
80\end{array}$ & $\begin{array}{l}\text { n? } \\
\text { ลิ }\end{array}$ & $\frac{n}{2}$ & $\frac{2}{a}$ & $\begin{array}{l}10 \\
8 \\
0\end{array}$ & $\begin{array}{l}n \\
i \\
0 \\
0\end{array}$ & $\frac{n}{6}$ & $\frac{2}{2}$ & $\begin{array}{l}n \\
8\end{array}$ & 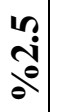 & $\frac{10}{6}$ & $\frac{\theta}{30}$ & $\begin{array}{l}10 \\
8\end{array}$ & $\stackrel{n}{i}$ & \\
\hline+ & + & - & - & - & - & - & - & - & - & - & - & + & + & $\begin{array}{l}+ \\
+\end{array}$ & - & + & $\begin{array}{l}+ \\
+\end{array}$ & $\begin{array}{l}+ \\
+\end{array}$ & + & اليوكالبتوس زيت \\
\hline - & + & - & - & + & - & - & - & - & - & - & - & - & - & - & - & - & - & - & - & زيت النيم \\
\hline
\end{tabular}

أظهرت النتائج ان زيت النيم لم يكن فعالا ضد الأحياء المجهريـة المختبرة برغم من وجود الدر اسـات تؤكد

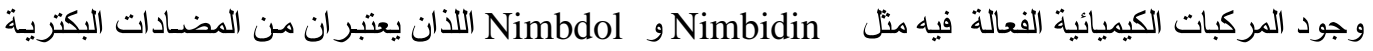

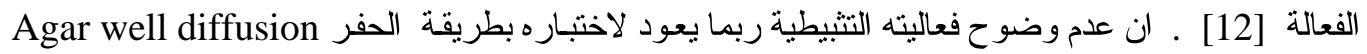

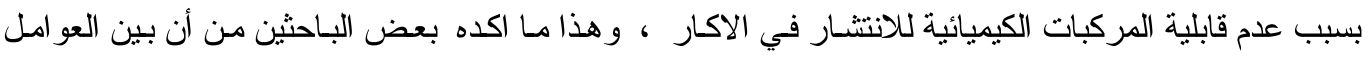

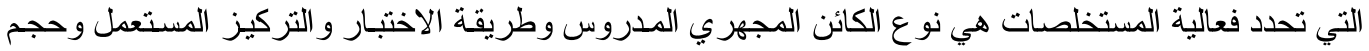

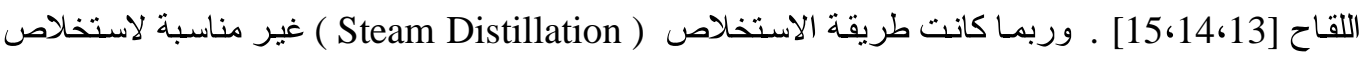

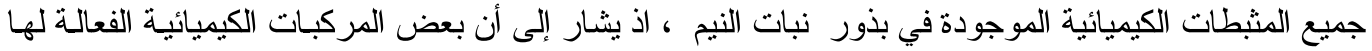

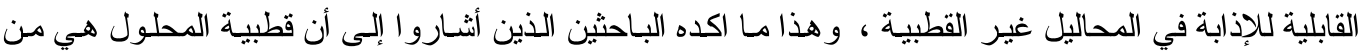

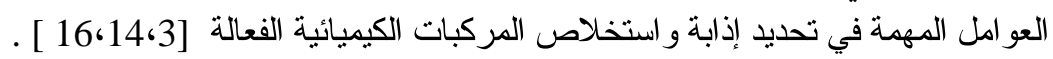

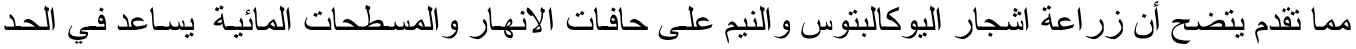

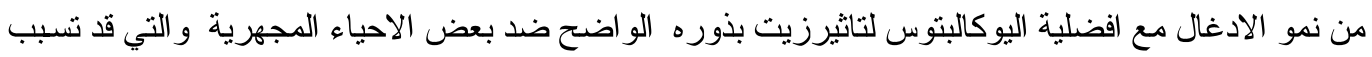

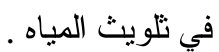

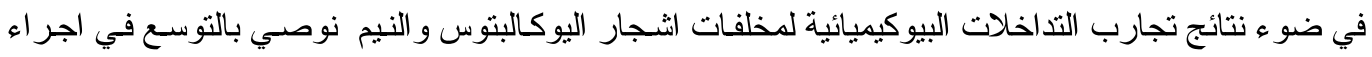

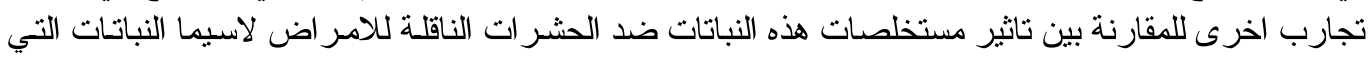

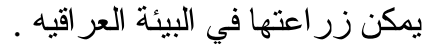

\section{المصادر}

1. Haig, T, Partley, J., and An, M.(1998) . Allelopathy: from concept to reality. Proceedings of the 9th Aust. Agro. Conference, Wagga Wagga

2. Mulla MS, Su T. Activity and biological effects of neem products against arthropods of medical and veterinary importance. Journal of the American Mosquito Control Association, 1999, 15(2): 133-52.

3. Mahasneh, A. M., Abbas, J. A. and El-Oqilah, A. A. 1996. Antimicrobial activity of extracts of herbal plants used in the traditional medicine of Bahrain. phytotherapy Res. 10: 253-257.

4. Reigosa,M.J.,Sanchez-Moreirars,A.,Gonzalez,L(1999).Ecophysiological Approach in Allelopathy In ciritical reviews in plant sciences. 18(5): 577 - 608.

5. Vanghn, S.F and Boydston, R.A. (1997). Volatile allelochemicals releases by crucifer green manures. Chem. J. Ecol. 23:2107 - 2116. 
6. Del-Moral, R. and Muller, C. H. 1970. The allelopathic effects of Eucalyptus camaldulensis . Am. Midl. Nat. 83: 254-282.

7. Rizvi, S. J. and Rizvi, V. 1992. Allelopathy. Basic and Applied Aspects. Chapman and Hall Press. London

8. Mariluz Sp. Dionglay1, Irma I. Palanginan1 and Maristar Sp. Dionglay. EVALUATION OF ANTIBACTERIAL ACTIVITES OF E. CAMALDULENSIS LEAF OIL AGAINST SCABIES. Fax: (049) 536 3630, Email: mariluz_dionglay@yahoo.com

9. Mulla MS. and Su T. 1999 .Activity and biological effects of neem products against arthropods of medical and veterinary importance. Journal of the American Mosquito Control Association, 15(2): 133-52.

10. Rice, E. L. 1984. Allelopathy. 2nd Ed, Academic press, New York.

11. Meliaceae A.Juss . 2005. Phonlic Acids in neem (Azadirachta indica) A major Pre-Existing Secondary Metabolites .J herb. Pharmacother . 5(1):35-43.

12. Obomanu F.G., Ogbalu K.O., U .U. Gabriel, G.K. Fekarurhobo and B.I. Adediran .2006.Larvicidal properties of Lepidagathis alopecuroides and Azadirachta indica on Anopheles gambiae and Culex quinquefasciatus .African Journal of Biotechnology .5(9):761-765 .

13. العاني، اوس هلال جاسم. 1998. در اسـة مكونات الحبـة السوداء المحلية.

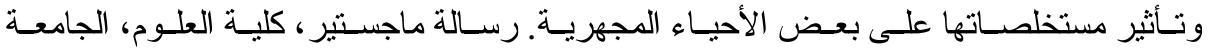

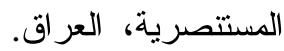

14. Cowan, M. M. 1999. Plant products as antimicrobial agents. Clin. Microbial. Rev., 12: 564-582.

15. المحدي، لهيب رجب عمـاد. 2002. عزل وتتخيص بكتريا Pseudomonas aeruginosa

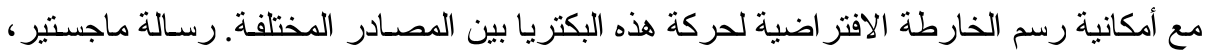

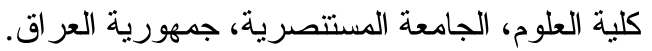

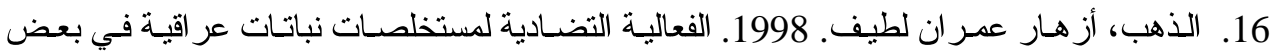
البكتريا الممرضة. أزهار عسرالة ماجستير ، كلية العلوم، جامعة بابل، جمهورية العر اق. 